\title{
High serum glucose levels are associated with a higher perceived age
}

\author{
Raymond Noordam • David A. Gunn • \\ Cyrena C. Tomlin • Andrea B. Maier • \\ Simon P. Mooijaart • P. Eline Slagboom • \\ Rudi G. J. Westendorp • Anton J. M. de Craen • \\ Diana van Heemst • \\ On behalf of the Leiden Longevity Study Group
}

Received: 13 August 2011 /Accepted: 25 October 2011/Published online: 20 November 2011

(C) The Author(s) 2011. This article is published with open access at Springerlink.com

\begin{abstract}
Estimating perceived age by facial photographs is a good estimate of health in elderly populations. Previously, we showed that familial longevity is marked by a more beneficial glucose metabolism already at middle age. As glucose is also related to skin aging, this study aimed to investigate the association between glucose metabolism and perceived age. Perceived age was assessed using facial photographs and non-fasted glucose and insulin were measured in 602 subjects from
\end{abstract}

Electronic supplementary material The online version of this article (doi:10.1007/s11357-011-9339-9) contains supplementary material, which is available to authorized users.

R. Noordam · A. B. Maier · S. P. Mooijaart •

R. G. J. Westendorp · A. J. M. de Craen •

D. van Heemst $(\bowtie)$

Department of Gerontology and Geriatrics,

Leiden University Medical Center,

PO Box 9600, 2300 RC Leiden, The Netherlands

e-mail: D.van_Heemst@lumc.nl

D. A. Gunn • C. C. Tomlin

Unilever Discover, Colworth House,

Sharnbrook, Bedfordshire, UK

P. E. Slagboom

Section of Molecular Epidemiology,

Department of Medical Statistics and Bioinformatics,

Leiden University Medical Center,

Leiden, the Netherlands

P. E. Slagboom • R. G. J. Westendorp

Netherlands Consortium of Healthy Aging (NCHA),

Leiden, The Netherlands the Leiden Longevity Study. Non-diabetic subjects ( $n=$ 569) were divided in three strata according to their glucose levels, and diabetic subjects $(n=33$; as a proxy of long-term hyperglycemic exposure) were included as a fourth stratum. Considered confounding factors were gender, chronological age, current smoking, body mass index, photo-damage score, and insulin levels. Perceived age was increased from 59.6 years $(\mathrm{SE}=0.3)$ in the first stratum to 61.2 years $(\mathrm{SE}=0.6)$ in diabetic subjects ( $p$ for trend $=0.002$ ). In non-diabetic subjects only, perceived age was increased from 59.6 years $(\mathrm{SE}=0.3)$ in the first stratum to 60.6 years $(\mathrm{SE}=0.3)$ in the third stratum ( $p$ for trend $=0.009)$. Continuously, perceived age increased 0.40 years $(\mathrm{SE}=0.14, p=0.006)$ per $1 \mathrm{mmol} / \mathrm{L}$ increase in glucose level in non-diabetic subjects. The present study demonstrates that, also among nondiabetic subjects, higher glucose levels are associated with a higher perceived age. Future research should be focused on elucidating possible mechanisms linking glucose levels to perceived age.

Keywords Perceived age · Serum glucose levels . Diabetes · Aging

\section{Introduction}

Perceived age, or estimated age, is routinely assessed by physicians to assess a patient's health condition. 
Perceived age was recently shown to be a robust clinical marker for assessment of (healthy) aging. Subjects looking old for their age had a greater risk of both morbidity and mortality (Christensen et al. 2009). Several intrinsic and extrinsic factors have been described to associate with perceived age. For example, sun exposure, smoking, and low body mass index associate with an older appearance, whereas a high body mass index and high social class associate with a younger appearance for one's age (Rexbye et al. 2006). Moreover, twin studies suggest also a genetic contribution to perceived age (Shekar et al. 2005; Gunn et al. 2009). Changes in facial features, as skin wrinkling (Gunn et al. 2009), skin color homogeneity (Matts et al. 2007), lip size (Gunn et al. 2009), and sag (Ozdemir et al. 2002) have all been linked to perceived age. However, it is largely unknown what biological processes underlie these facial aging features.

Glucose levels have been proposed to be a major determinant of the aging process. Many age-related diseases occur earlier in life, with a higher incidence in diabetic patients compared to healthy controls (Cerami 1985; Seshasai et al. 2011). The hypothesis that an increased glucose level acts as an "aging accelerator" has been supported by several research findings in both lower model organisms and humans. In lower model organisms, genetic alterations in the insulin and insulin-like growth factor signaling pathway significantly increased lifespan (Bartke 2008; Lakowski and Hekimi 1996). Long-lived mammalian models often display higher insulin sensitivity in conjunction with low levels of insulin and glucose, making it difficult to disentangle the exact contribution of each (Bartke 2008). We have recently shown in humans, participating in the Leiden Longevity Study, that offspring from long-lived families, at middle age, have a lower prevalence of diabetes and cardiovascular disease compared to controls of comparable age (Westendorp et al. 2009). Moreover, both fasted and non-fasted glucose levels as well as fasted insulin levels were lower in offspring from long-lived families compared to controls (Rozing et al. 2010, 2009). Offspring and partners did not differ in the number of single nucleotide polymorphisms associated with decreased beta cell function (Beekman et al. 2010; Mooijaart et al. 2011). However, using hyperinsulinemic euglycemic clamp studies, we showed that the lower glucose levels are, at least in part, explained by a higher peripheral insulin sensitivity in the offspring group (Wijsman et al. 2011).

Skin aging has also been linked to glucose levels. For example, it has been shown that extensive exposure to hyperglycemic conditions causes premature cellular senescence in human skin fibroblasts (Blazer et al. 2002; Dekker et al. 2009). Furthermore, diabetic patients display a higher degree of cross-links in their collagen, which is assumed to be mainly caused by advanced glycation end products (AGEs), an end-stage waste product of exposure to high glucose levels (Paul and Bailey 1996). The crosslinks in collagen prevent efficient repair, which in turn will cause premature aging of the skin. Aging of the skin is a major component of facial aging and is strongly associated with perceived age (Gunn et al. 2009). Together with the potential role of glucose in longevity, these findings have led to the hypothesis that higher levels of non-fasted glucose, as a proxy of higher average blood glucose during a day, may lead to a higher perceived age. In this study, we assessed this hypothesis by analyzing the association of nonfasted glucose levels with perceived age in a cohort of 602 middle-aged subjects.

\section{Methods}

Study population

The Leiden Longevity Study was designed to identify genetic and phenotypic markers related to longevity. A total of 421 families were recruited consisting of long-lived Caucasian siblings together with their offspring and partners thereof. Inclusion was only performed when at least two long-lived siblings were still alive and fulfilled the age criteria of 89 years in case of males and 91 years for females (Schoenmaker et al. 2006). The siblings were not selected on health conditions or demographics. Because proper controls at high age are lacking, the offspring from these nonagenarian siblings were asked to participate and serve as cases, because they have an increased propensity to reach an old age. The partners of the offspring were asked to participate in the study as controls of comparable age.

From the group of offspring and their partners, a subpopulation of 669 participants was asked to 
participate in this cross-sectional study. As the association between glucose and perceived age was found to be similar in the offspring group compared to their partners, we treated the study population as being one. Data from the non-fasted blood samples (glucose and insulin) were missing from 30 subjects. Photo-damage scoring could not be determined in male subjects with beards $(n=37)$. Hence, in total, 602 subjects (including 33 diabetic subjects) were used for the analyses to assess the association between glucose levels and perceived age. Subjects were regarded as having diabetes if they had a nonfasted glucose level $>11.0 \mathrm{mmol} / \mathrm{L}$, a previous medical history of diabetes, and/or used glucoselowering agents. The Leiden Longevity Study was approved by the Medical Ethical Committee of the Leiden University Medical Center, and informed consent was obtained from all subjects.

\section{Blood measurements}

Fully automated equipment was used to perform the serum measurements. Glucose levels were measured with the Hitachi Modular P800 (Roche, Almere, the Netherlands). Insulin levels were measured using Immulite 2500 (DPC, Los Angeles, CA, USA). Coefficients of variance of both measurements were below $5 \%$.

\section{Data collection}

The method for assessing a person's perceived age has been described and validated previously (Christensen et al. 2009; Gunn et al. 2008, 2009). In short, subjects were asked to come to the study center without any makeup or hairstyling products. From every participant, one photograph from the front and one at $45^{\circ}$ were taken. Photographs, with concealed hair and clothing, were assessed by 60 independent assessors. Assessors were predominately British and had no previous age assessment experience. There was some variation in the age and gender between the assessors. However, it was previously shown that the assessors' age and gender have a negligible effect on the mean age assessment when a large number of assessors are used (Gunn et al. 2008). The mean age assessment of all 60 independent assessments is herein called the perceived age. Besides determining the perceived age, images were also assessed by two independent dermatologists for photo-damage scoring (Griffiths et al. 1992), in which a higher score means a higher sun-induced damage.

Additionally, lifestyle questionnaires were sent to the subjects to obtain insights in their smoking habits. Weight and height, for calculating the body mass index, were measured by research nurses at the study center.

Statistical analyses

Body mass index was calculated by dividing weight (in kilograms) with height (in meters) squared. Insulin levels were not normally distributed and therefore $\log$ transformed. In the dataset, data on smoking were dichotomized in current smokers and non-smokers.

For examination of the association between glucose levels and perceived age, we used linear regression models. The first two analyses were performed in the total group $(n=602)$ comprising the non-diabetic subjects $(n=569)$, divided over three strata dependent on their glucose levels, as well as the diabetic subjects $(n=33)$, which were included as a fourth stratum. First, we assessed the association between perceived age dependent on glucose strata in a model which was only adjusted for gender. In a second analysis, we additionally adjusted for body mass index, current smoking, and photo-damage score, which were considered confounding factors. Both analyses were repeated in non-diabetic subjects $(n=569)$ only, which were divided over three strata dependent on their glucose levels. Subsequently, we tested both in the total group $(n=602)$ as well as in the subgroup comprising the non-diabetic subjects only $(n=569)$ whether the observed association between glucose and perceived age was independent of insulin levels. For this, logtransformed insulin levels were included as an additional independent variable in the model. This last analysis was repeated with glucose as a continuous variable instead of strata to assess the influence of $1 \mathrm{mmol} / \mathrm{L}$ increase in glucose level on perceived age.

All statistical analyses were performed using the SPSS program for Windows (version 17, USA). The $p$ values below 0.05 were considered to be statistically significant. 


\section{Results}

Characteristics of the study population

General characteristics of the study population are presented in Table 1. Compared to non-diabetic subjects, diabetic subjects tended to have, on average, a higher chronological age, a lower percentage of females, a lower photo-damage score, and a higher body mass index. Moreover, diabetic subjects had higher levels of non-fasted insulin compared to nondiabetic subjects.

Association of glucose levels and perceived age

The associations between serum glucose levels and perceived age are presented in Table 2 and Fig. 1. The associations between serum glucose levels and perceived age were assessed in the combined group of non-diabetic subjects, which were divided over three strata dependent on their glucose levels and diabetic subjects, which latter were added as a fourth stratum as well as in the group of non-diabetic subjects only. Analyses were performed with adjustment for gender only, as well as with additional adjustment for chronological age, body mass index, smoking, and photo-damage score. The associations of the considered confounding factors with glucose levels and perceived age are presented in supplementary Table 1. In the combined group of diabetic and non-diabetic subjects, using a model only adjusted for gender, higher levels of glucose were associated with a higher perceived age $\left(p_{\text {trend }}=0.001\right)$. This association persisted when the analysis was restricted to non-diabetic subjects only $\left(p_{\text {trend }}=0.001\right)$. Using a model adjusted for chronological age, gender, body mass index, current smoking, and photo-damage, perceived age was increased from 59.7 years in the first stratum of non-diabetic subjects to 61.1 years in the stratum of the diabetic subjects $\left(p_{\text {trend }}=0.003\right)$. In non-diabetic subjects only, perceived age was increased from 59.7 years in the first stratum to 60.4 years in the third stratum $\left(p_{\text {trend }}=0.045\right)$. To test whether this association was independent from insulin levels, the previous model was additionally adjusted for insulin levels. As presented in Fig. 1, the association between glucose and perceived age persisted across the combined group of non-diabetic and diabetic subjects, as well is in the group of non-diabetic subjects only.

Using the fully adjusted model with glucose levels from non-diabetic subjects as a continuous variable instead of tertiles, the increase of perceived age per $1 \mathrm{mmol} / \mathrm{L}$ increase of glucose was approximately 0.40 years $(95 \%$ CI $0.12-0.69, p=0.006)$. The association of glucose levels and perceived age was similar in males and females (results not shown). Additionally, the association between glucose levels and perceived age was similar for offspring from long-lived families and their partners (results not shown).

\section{Discussion}

The main goal of this study was to assess the association between glucose levels and perceived age. We showed that diabetic subjects had a tendency toward higher perceived age compared to non-

Table 1 Characteristics of the study population

\begin{tabular}{lllll}
\hline & \multicolumn{2}{l}{ Strata of glucose levels in non-diabetic subjects } & & Diabetic subjects \\
\cline { 2 - 4 } & Low $(N=191)$ & Medium $(N=192)$ & High $(N=186)$ & $(N=33)$ \\
\hline Glucose range (mmol/L) & $3.3-5.2$ & $5.3-6.1$ & $6.2-10.5$ & $4.4-26.3$ \\
Chronological age (years) & $61.8(6.7)$ & $63.4(6.5)$ & $63.4(6.9)$ & $64.8(6.1)$ \\
Females, no. (\%) & $107(56.0)$ & $98(51.0)$ & $100(53.8)$ & $15(45.5)$ \\
Photo-damage score (points) & $4.51(1.2)$ & $4.68(1.3)$ & $4.84(1.3)$ & $4.36(1.2)$ \\
Current smoking, no. $(\%)$ & $24(12.6)$ & $20(10.4)$ & $29(15.6)$ & $4(12.1)$ \\
Body mass index $\left(\mathrm{kg} / \mathrm{m}^{2}\right)$ & $25.8(3.3)$ & $26.9(4.1)$ & $26.6(4.2)$ & $29.7(5.2)$ \\
Ln (insulin) $(\mathrm{mU} / \mathrm{L})$ & $2.44(0.8)$ & $2.75(0.7)$ & $3.31(0.7)$ & $3.39(0.7)$ \\
\hline
\end{tabular}

All parameters are presented as mean with standard deviation unless indicated otherwise 
Table 2 Association of glucose levels and perceived age

\begin{tabular}{|c|c|c|c|c|c|c|}
\hline & \multicolumn{4}{|c|}{ Strata of glucose levels in non-diabetic subjects } & \multirow{2}{*}{$\begin{array}{l}\text { Diabetic subjects } \\
(N=33)\end{array}$} & \multirow[b]{2}{*}{$P_{\text {trend }} \mathrm{b}$} \\
\hline & Low $(N=191)$ & Medium $(N=192)$ & $\operatorname{High}(N=186)$ & $P_{\text {trend }}{ }^{\mathrm{a}}$ & & \\
\hline Perceived age (years) ${ }^{\mathrm{c}}$ & $58.0(56.9-59.1)$ & $59.8(58.7-60.9)$ & $60.8(59.6-61.9)$ & 0.001 & $60.2(57.5-62.8)$ & 0.001 \\
\hline Perceived age (years) ${ }^{\mathrm{d}}$ & $59.7(59.2-60.3)$ & $60.2(59.6-60.8)$ & $60.4(59.9-61.0)$ & 0.045 & $61.1(59.9-62.3)$ & 0.003 \\
\hline
\end{tabular}

All presented results are means of perceived age with $95 \%$ confidence interval

${ }^{\text {a }} P$ for trends were calculated over the three strata of glucose levels in non-diabetic subjects

${ }^{\mathrm{b}} P$ for trends were calculated over the three strata of glucose in non-diabetic subjects and diabetic subjects as a fourth stratum

${ }^{\mathrm{c}}$ Adjusted for gender

${ }^{\mathrm{d}}$ Adjusted for gender, chronological age, body mass index, current smoking, and photo-damage

diabetic subjects. Moreover, also in non-diabetic subjects, higher glucose levels were associated with a higher perceived age, independent from confounding factors. Taken together, these results suggest that exposure to elevated glucose levels may indeed cause premature aging of the skin.

The presented study has a few shortcomings to address. The cross-sectional study design used makes it impossible to infer a causal relationship between glucose and perceived age. In addition, glucose levels

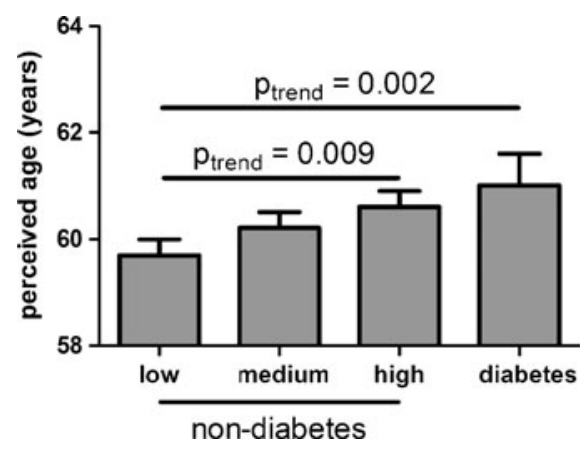

Fig. 1 Association of glucose levels with perceived age. The figure depicts the association between glucose level and perceived age $(n=602)$. Non-diabetic subjects $(n=569)$ were divided over tertiles of glucose and diabetic subjects $(n=33)$ included as a fourth category. Mean perceived age and obtained $p$ values were adjusted for gender, chronological age, body mass index, current smoking, photo-damage score, and Ln (insulin) level as described in "Methods." For the non-diabetic subjects, the mean perceived age (SE) was 59.6 years $(0.3)$ in the low, 60.2 years $(0.3)$ in the medium, and 60.6 years $(0.3)$ in the high glucose tertile. For the diabetic subjects, the mean perceived age (SE) was 61.2 years (0.6). In a model using only the non-diabetic subjects, we observed a statistical significant positive trend $\left(p_{\text {trend }}=0.009\right)$ for the increase in perceived age dependent on glucose tertiles. Inclusion of the diabetic subjects in the model also revealed a highly significant positive trend $\left(p_{\text {trend }}=0.002\right)$ were measured in non-fasted samples, as a proxy of the average serum glucose level over the day, which could have resulted in an increased variation in the data due to variation in time since the last meal. However, the resulting non-differential misclassification has probably resulted in a weaker association between glucose levels and perceived age. Another point to address is our earlier observation that offspring from nonagenarian siblings displayed a lower prevalence of type 2 diabetes and, moreover, displayed lower levels of glucose (Rozing et al. 2010, 2009). However, we observed a similar association between glucose and perceived age in offspring and partners (results not shown). For this reason, we treated these two groups as being one. Despite these shortcomings, the significance of the results and the robustness of the analyses support the hypothesis that glucose level might be involved in skin aging.

Skin aging is a major component of facial aging and is strongly associated with perceived age (Gunn et al. 2009). Several mechanisms have been described through which glucose could contribute to the skin aging process. A first option is via the formation of AGEs. An initial reaction (the Maillard reaction) of glucose with an amino acid (e.g., lysine) forms pentosidine, which is an AGE. This newly formed molecule is able to react with lysine and arginine residues on collagen molecules causing cross-links between collagen molecules (Paul and Bailey 1996). With an increasing concentration of AGEs in the skin, an increasing number of cross-links are formed. This phenomenon eventually prevents efficient collagen repair, which in turn results in premature aging of the skin (Avery and Bailey 2006; Paul and Bailey 1996). Moreover, not only collagen, but also elastin, is 
affected by AGEs, resulting in a reduction of skin elasticity. Importantly, AGEs are not only affecting skin tissue but also other collagenous tissues throughout the body and as such may contribute to cardiovascular disease (Simm et al. 2007), reduced kidney function (Yan et al. 2007a), and vascular complications in diabetes (Yan et al. 2007b). Because AGEs were shown to be associated with pathological conditions, we hypothesized that AGEs could be inferred to the previous observed association between perceived age and morbidities (Christensen et al. 2009). Measurements of skin fluorescence (the formed cross-links in collagen are fluorescent) or circulating AGEs (e.g., circulating pentosidine) could give us more insights in whether glucose levels are causally linked to perceived age via AGEs.

A second possibility through which serum glucose levels may cause an older-looking appearance is the induction of premature cellular senescence. Several studies have shown that culturing human fibroblasts under hyperglycemic conditions results in both an increased amount of reactive oxygen species at a cellular level as well as an increased induction of premature cellular senescence (Blazer et al. 2002; Chintapalli et al. 2007; Dekker et al. 2009). Moreover, it has been shown, by measuring p16-positive cells, that the number of senescent cells in human skin increases with age (Ressler et al. 2006). Histological examination of the number of p16-positive cells or other markers of cellular senescence could give us a hint whether long-term exposure to, on average, higher glucose levels might result in a higher number of senescent cells which in turn may cause premature skin aging and a higher perceived age.

In conclusion, our data show that higher glucose levels were associated with higher perceived age, independently from confounding factors. More research has to be performed to determine whether glucose is a causal factor behind an increased perceived age and, if so, through which possible mechanisms (AGEs, premature induced cellular senescence, or others) glucose could promote skin aging.

Acknowledgments We would like to thank all participants, the secretary staff (Meriam H.G.F. van der Star and Ellen H.M. Bemer-Oorschot), and the research nurse (Corrie Groenendijk) for their valuable contribution to this study. We would also like to thank Chris Griffiths, Sharon Catt, Tamara Griffiths, and Stephanie Ogden for generating the photo-damage scores and Peter Murray for calculating the mean perceived ages. This study was funded by the Innovation Oriented Research Program on Genomics (SenterNovem; IGE01014 and IGE5007), the Centre for Medical Systems Biology (CMSB), the Netherlands Genomics Initiative/Netherlands Organization for scientific research (NGI/NWO; 05040202 and 050-060-810. NCHA), Unilever PLC, and the EU-funded Network of Excellence Lifespan (FP6 036894). P. Eline Slagboom is supported by an unrestricted grant from the Netherlands Genomics Initiative (NCHA 050-060-810).

Open Access This article is distributed under the terms of the Creative Commons Attribution Noncommercial License which permits any noncommercial use, distribution, and reproduction in any medium, provided the original author(s) and source are credited.

\section{References}

Avery NC, Bailey AJ (2006) The effects of the Maillard reaction on the physical properties and cell interactions of collagen. Pathol Biol (Paris) 54(7):387-395

Bartke A (2008) Impact of reduced insulin-like growth factor-1/ insulin signaling on aging in mammals: novel findings. Aging Cell 7(3):285-290

Beekman M, Nederstigt C, Suchiman HE, Kremer D, van der Breggen R, Lakenberg N, Alemayehu WG, de Craen AJ, Westendorp RG, Boomsma DI, de Geus EJ, HouwingDuistermaat JJ, Heijmans BT, Slagboom PE (2010) Genome-wide association study (GWAS)-identified disease risk alleles do not compromise human longevity. Proc Natl Acad Sci USA 107(42):18046-18049

Blazer S, Khankin E, Segev Y, Ofir R, Yalon-Hacohen M, Kra-Oz Z, Gottfried Y, Larisch S, Skorecki KL (2002) High glucose-induced replicative senescence: point of no return and effect of telomerase. Biochem Biophys Res Commun 296(1):93-101

Cerami A (1985) Hypothesis. Glucose as a mediator of aging. J Am Geriatr Soc 33(9):626-634

Chintapalli J, Yang S, Opawumi D, Goyal SR, Shamsuddin N, Malhotra A, Reiss K, Meggs LG (2007) Inhibition of wild-type p66ShcA in mesangial cells prevents glycooxidant-dependent FOXO3a regulation and promotes the survival phenotype. Am J Physiol Renal Physiol 292 (2):F523-F530

Christensen K, Thinggaard M, McGue M, Rexbye H, Hjelmborg JV, Aviv A, Gunn D, van der Ouderaa F, Vaupel JW (2009) Perceived age as clinically useful biomarker of ageing: cohort study. BMJ 339:b5262

Dekker P, Maier AB, van Heemst D, de Koning-Treurniet C, Blom J, Dirks RW, Tanke HJ, Westendorp RG (2009) Stress-induced responses of human skin fibroblasts in vitro reflect human longevity. Aging Cell 8(5):595-603

Griffiths CE, Wang TS, Hamilton TA, Voorhees JJ, Ellis CN (1992) A photonumeric scale for the assessment of cutaneous photodamage. Arch Dermatol 128(3):347-351

Gunn DA, Murray PG, Tomlin CC, Rexbye H, Christensen K, Mayes AE (2008) Perceived age as a biomarker of ageing: a clinical methodology. Biogerontology 9 (5):357-364 
Gunn DA, Rexbye H, Griffiths CE, Murray PG, Fereday A, Catt SD, Tomlin CC, Strongitharm BH, Perrett DI, Catt M, Mayes AE, Messenger AG, Green MR, van der Ouderaa F, Vaupel JW, Christensen K (2009) Why some women look young for their age. PLoS One 4(12):e8021

Lakowski B, Hekimi S (1996) Determination of life-span in Caenorhabditis elegans by four clock genes. Science 272 (5264):1010-1013

Matts PJ, Fink B, Grammer K, Burquest M (2007) Color homogeneity and visual perception of age, health, and attractiveness of female facial skin. J Am Acad Dermatol 57(6):977-984

Mooijaart SP, van Heemst D, Noordam R, Rozing MP, Wijsman CA, de Craen AJ, Westendorp RG, Beekman M, Slagboom PE (2011) Polymorphisms associated with type 2 diabetes in familial longevity: The Leiden Longevity Study. Aging 3:55-62

Ozdemir R, Kilinc H, Unlu RE, Uysal AC, Sensoz O, Baran $\mathrm{CN}$ (2002) Anatomicohistologic study of the retaining ligaments of the face and use in face lift: retaining ligament correction and SMAS plication. Plast Reconstr Surg 110(4):1134-1147, discussion 1148-1139

Paul RG, Bailey AJ (1996) Glycation of collagen: the basis of its central role in the late complications of ageing and diabetes. Int J Biochem Cell Biol 28(12):1297-1310

Ressler S, Bartkova J, Niederegger H, Bartek J, ScharffetterKochanek K, Jansen-Durr P, Wlaschek M (2006) p16INK4A is a robust in vivo biomarker of cellular aging in human skin. Aging Cell 5(5):379-389

Rexbye H, Petersen I, Johansens M, Klitkou L, Jeune B, Christensen K (2006) Influence of environmental factors on facial ageing. Age Ageing 35(2):110-115

Rozing MP, Westendorp RG, de Craen AJ, Frolich M, de Goeij MC, Heijmans BT, Beekman M, Wijsman CA, Mooijaart SP, Blauw GJ, Slagboom PE, van Heemst D (2010) Favorable glucose tolerance and lower prevalence of metabolic syndrome in offspring without diabetes mellitus of nonagenarian siblings: the Leiden longevity study. J Am Geriatr Soc 58(3):564-569

Rozing MP, Westendorp RG, Frolich M, de Craen AJ, Beekman M, Heijmans BT, Mooijaart SP, Blauw GJ, Slagboom PE, van Heemst D (2009) Human insulin/IGF-1 and familial longevity at middle age. Aging (Albany NY) 1(8):714-722

Schoenmaker M, de Craen AJ, de Meijer PH, Beekman M, Blauw GJ, Slagboom PE, Westendorp RG (2006) Evidence of genetic enrichment for exceptional survival using a family approach: the Leiden Longevity Study. Eur J Hum Genet 14(1):79-84

Seshasai SR, Kaptoge S, Thompson A, Di Angelantonio E, Gao P, Sarwar N, Whincup PH, Mukamal KJ, Gillum RF, Holme I, Njolstad I, Fletcher A, Nilsson P, Lewington S, Collins R, Gudnason V, Thompson SG, Sattar N, Selvin E, $\mathrm{Hu}$ FB, Danesh J (2011) Diabetes mellitus, fasting glucose, and risk of cause-specific death. N Engl J Med 364(9):829-841

Shekar SN, Luciano M, Duffy DL, Martin NG (2005) Genetic and environmental influences on skin pattern deterioration. J Invest Dermatol 125(6):1119-1129

Simm A, Wagner J, Gursinsky T, Nass N, Friedrich I, Schinzel R, Czeslik E, Silber RE, Scheubel RJ (2007) Advanced glycation endproducts: a biomarker for age as an outcome predictor after cardiac surgery? Exp Gerontol 42(7):668675

Westendorp RG, van Heemst D, Rozing MP, Frolich M, Mooijaart SP, Blauw GJ, Beekman M, Heijmans BT, de Craen AJ, Slagboom PE (2009) Nonagenarian siblings and their offspring display lower risk of mortality and morbidity than sporadic nonagenarians: The Leiden Longevity Study. J Am Geriatr Soc 57(9):1634-1637

Wijsman CA, Rozing MP, Streefland TC, le Cessie S, Mooijaart SP, Slagboom PE, Westendorp RG, Pijl H, van Heemst D (2011) Familial longevity is marked by enhanced insulin sensitivity. Aging Cell 10(1):114-121

Yan HD, Li XZ, Xie JM, Li M (2007a) Effects of advanced glycation end products on renal fibrosis and oxidative stress in cultured NRK-49F cells. Chin Med J (Engl) 120 (9):787-793

Yan SF, D'Agati V, Schmidt AM, Ramasamy R (2007b) Receptor for Advanced Glycation Endproducts (RAGE): a formidable force in the pathogenesis of the cardiovascular complications of diabetes \& aging. Curr Mol Med 7 (8):699-710 